\title{
Marketing Support Trials
}

National Cancer Institute

\section{Source}

National Cancer Institute. Marketing Support Trials. NCI Thesaurus. Code C142603.

A type of clinical trial in which the therapeutic benefits of a marketed product are either clarified or preferentially compared to another marketed product. 